\title{
On the Vortex Flow in Bounded Domains
}

\author{
D. Dürr ${ }^{1 \star}$ and M. Pulvirenti ${ }^{2 \star \star}$
}

1 Hill Center for the Math. Sciences, Rutgers University, New Brunswick, NJ 08903, USA

2 Istituto Matematico, Universita di Roma, Roma, Italy

\begin{abstract}
We consider the motion of $N$ vortices in bounded domains in $\mathbb{R}^{2}$. We prove that the set of initial positions which lead to a collapse of two or more vortices has Lebesgue measure zero. We extend this result to the stochastic motion of the vortices, where the stochasticity comes from a Wiener-noise term, which is added to the deterministic equation of motion.
\end{abstract}

\section{Introduction}

A system of $N$ vortices ( $x_{i} \in D$ is the position of the $i^{\text {th }}$ vortex and $\gamma_{i} \in \mathbb{R}$ its vorticity) in a domain $D \subseteq \mathbb{R}^{2}$ satisfies the following equations of motion

$$
\dot{x}_{i}(t)=\sum_{\substack{j=1 \\ j \neq i}}^{N} \gamma_{j} K\left(x_{i}(t), x_{j}(t)\right), \quad i=1, \ldots, N,
$$

with

$$
K(x, y)=\left(\nabla_{x}^{\perp} g\right)(x, y) ; \quad x, y \in D,
$$

$g$ being the fundamental solution of the Poisson equation with the appropriate boundary conditions, $\nabla^{\perp}=\left(\partial_{2},-\partial_{1}\right)$, and $\partial_{k}, k=1,2$ is the partial derivative with respect to the $k^{\text {th }}$ component.

The evolution equation (1.1) is equivalent to the Euler equation (except for an infinity constant due to the self-energy factor) and describes the dynamics of an incompressible fluid in which the vorticity is sharply concentrated around the points $x_{i}$. This model was introduced by Kirchhoff [1]. See also [2] for a precise connection with the Euler equation.

The following three choices of the domain $D$ are of interest for physics.

(i) $D=\mathbb{R}^{2}$, i.e. $g(x, y)=-\frac{1}{2 \pi} \ln (|x-y|)$.

* On leave of the Fachbereich Mathematik, RUB, 4630 Bochum, Federal Republic of Germany. Supported by a DFG-fellowship

$\star \star$ Partially supported by Italian CNR 
(ii) $D=\bar{T}^{2}=[-L, L]^{2}$, i.e. $g$ is the Green's function of the Poisson equation with periodic boundary conditions.

(iii) $D$ is a bounded domain with smooth boundary, and $g$ is the Green's function for Dirichlet boundary conditions. In this case the velocity field at the boundary $\partial D$ is tangent to $\partial D$, i.e. for $x_{i}=r \in \partial D$ and $n(r)$, the normal to $\partial D$ in $r$,

$$
\dot{x}_{i}=\sum_{j \neq i}^{N} \gamma_{j} K\left(r, x_{j}\right) \cdot n(r)=0,
$$

and this corresponds to the natural boundary conditions for the Euler equation.

Note that Eq. (1.1) has a hamiltonian structure in which the conjugate variables are the coordinates of the vortice's positions. Hence the following functions.

$$
\begin{aligned}
& H=\sum_{i \neq j} \gamma_{i} \gamma_{j} g\left(x_{i}, x_{j}\right) \quad \text { (energy), } \\
& M=\sum_{i=1}^{N} \gamma_{i} x_{i} \quad \text { (baricenter), } \\
& I=\sum_{i=1}^{N} \gamma_{i} x_{i}^{2} \quad \text { (moment of inertia) }
\end{aligned}
$$

are formal constants of the motion.

The typical logarithmic divergence of $g$ makes the construction of a flow satisfying (1.1) problematic. In the case in which $\gamma_{i}>0$ for all $i$, it is easily seen that the above first integrals preclude the development of singularities for the solutions, but in general collapses between vortices do appear for suitable initial conditions [3].

In this paper we prove that if $D$ is bounded [i.e. in cases (ii) and (iii)] the probability (with respect to the normalized Lebesgue measure on $D$ ) of finding collapses of two or more vortices in a finite amount of time is zero. Denoting by " $\varepsilon$-collapse" the event in which two or more vortices are closer than $\varepsilon$, we prove that the probability of an $\varepsilon$-collapse goes to zero as $\varepsilon$ goes to zero. This implies then the existence of an almost everywhere defined global flow satisfying (1.1).

The problem of constructing a flow defined almost everywhere for singular vector fields is treated in [4] in a general setup. Unfortunately these results (also valid only if the set of the singularities is compact) do not apply to the case considered here, because our vector field is only $L_{2-\varepsilon}$ locally.

The method presented here depends crucially on cancellations which are particular to our model.

The second problem we consider is the existence and uniqueness of the diffusion process in $\bar{T}^{2}$ governed by a stochastic differential equation with a drift term given by the right hand side of (1.1) and a diffusion constant $\beta$. This process is physically interesting because of its connection to the Navier Stokes equation [2]. We only consider periodic boundary conditions. The consideration of bounded domains $D$ with physical boundaries is more complicated: One has to describe the production of vorticity on the boundary which is necessary to restore the right boundary conditions for a viscous fluid, i.e. for $x_{i}=r \in \partial D$,

$$
\sum_{j \neq i} \gamma_{j} K\left(r, x_{j}\right)=0
$$


This case, as well as the case where the domain $D$ is unbounded (both for the deterministic and stochastic dynamics), seem to be more involved and presumably require different techniques than the ones used in this paper.

\section{Deterministic Evolution}

Let us consider a two dimensional flat torus $\bar{T}^{2}=[-L, L]^{2}, L>0$, and the evolution equation (1.1) with Green's function $g$ belonging to periodic boundary conditions, i.e.

$$
g(x, y)=-\frac{1}{(2 \pi L)^{2}} \sum_{\substack{k \in \mathbb{Z}^{2} \\ k \neq 0}} \pm \exp \left\{i \frac{2 \pi}{L} k \cdot(x-y)\right\}
$$

Remark. We note that in the case $D=\bar{T}^{2}$ a correct fluid dynamical interpretation would assume $\sum_{i=1}^{N} \gamma_{i} \equiv V=0 . V$ is the total vorticity of the fluid and since the velocity field is periodic, $V$ has to be zero by the circulation theorem. The condition $V=0$ combined with the incompressibility hypothesis and the continuity equation allows reconstruction of the velocity field from the vorticity field (up to a constant field determined by the initial conditions). This also shows the formal analogy between the Euler equation and the initial value problem (1.1). Nevertheless beyond the physical interpretation the evolution problem (1.1) in $\bar{T}^{2}$ makes sense also when $V \neq 0$. We consider this more general case.

Let $g_{\varepsilon}$ be any $C^{2}\left(\mathbb{R}^{2} \times \mathbb{R}^{2}\right)$ periodic and symmetric function (of periodicity $2 L$ ) such that for any $\varepsilon>0$

$$
\begin{gathered}
g_{\varepsilon}(x, y)=g(x, y) \quad \text { if } \quad|x-y|>\varepsilon, \\
\left|\nabla_{x} g_{\varepsilon}(x, y)\right| \leqq\left|\nabla_{x} g(x, y)\right| \leqq \frac{C}{|x-y|}
\end{gathered}
$$

for some constant $C>0$ and define

$$
K_{\varepsilon}=\nabla^{\perp} g_{\varepsilon}
$$

Let $\bar{T}^{2 N} \ni x \rightarrow x^{\varepsilon}(t)=S_{t}^{\varepsilon} x \in \bar{T}^{2 N}$ be the solution of the following initial value problem

$$
\begin{aligned}
\dot{x}_{i}^{\varepsilon}(t) & =\sum_{\substack{j=1 \\
j \neq i}}^{N} \gamma_{j} K_{\varepsilon}\left(x_{i}^{\varepsilon}(t), x_{j}^{\varepsilon}(t)\right), \\
x_{i}^{\varepsilon}(0) & =x_{i} ; \quad i=1, \ldots, N, \\
x & =\left(x_{1}, \ldots, x_{N}\right) ; \quad x^{\varepsilon}(t)=\left(x_{1}^{\varepsilon}(t), \ldots, x_{N}^{\varepsilon}(t)\right) .
\end{aligned}
$$

Since $K_{\varepsilon}$ is periodic the flow $S_{t}^{\varepsilon} x=x^{\varepsilon}(t)$ is well defined in $\bar{T}^{2}$ and $\nabla \cdot K_{\varepsilon}=0$ ensures the conservation of the Lebesgue measure on $\bar{T}^{2}$.

For (1.1) we can define a local flow $S_{t}$ up to the time of the first collision of two or more vortices. We denote by $\lambda$ the normalized Lebesgue measure on $\bar{T}^{2 N}$, i.e. 
$\lambda(d x)=(2 L)^{-N} d x_{1} \ldots d x_{N}$. We define the collision time $\tau$ as

$$
\begin{aligned}
& \tau(x)=\sup \left\{t \mid S_{t} x \notin N_{0}\right\}, \quad \text { if } \quad x \notin N_{0} \text {, } \\
& =0, \quad \text { if } x \in N_{0} \text {, }
\end{aligned}
$$

where $N_{0}=\left\{x \mid x_{i}=x_{j}\right.$ for some $\left.i \neq j\right\}$. We write for $t \leqq \tau(x) S_{t} x=\left(x_{1}(t), \ldots, x_{N}(t)\right)$. The following theorem says that the set of initial conditions which lead to collisions between vortices (collapses) has Lebesgue measure zero.

Theorem 2.1. For any $T>0$ there exists a set $N_{T} \subset \bar{T}^{2 N}$ such that

$$
\begin{gathered}
\lambda\left(N_{T}\right)=0, \\
\delta(x)=\min (\tau(x), T)=T \quad \text { if } \quad x \in \bar{T}^{2 N} \backslash N_{T} .
\end{gathered}
$$

Moreover let

$$
d_{T}(x)=\min _{i \neq j} \inf _{t \in[0, T]}\left|x_{i}(t)-x_{j}(t)\right|,
$$

then for $0<\varepsilon<1$

$$
\lambda\left(\left\{x \mid d_{T}(x)<\varepsilon\right\}\right) \leqq \frac{A(T+1)}{-\ln \varepsilon}
$$

for some constant $A>0$.

Proof. We set

$$
\begin{aligned}
\tau_{\varepsilon}(x) & =\min _{i \neq j} \sup _{t \in[0, T]}\left\{t|| x_{i}(t)-x_{j}(t) \mid>\varepsilon ; i \neq j\right\}, \\
& =0, \text { if }\left|x_{i}-x_{j}\right| \leqq \varepsilon \text { for some } i \neq j,
\end{aligned}
$$

and

$$
\delta_{\varepsilon}(x)=\min \left(T, \tau_{\varepsilon}(x)\right) .
$$

Clearly

$$
\lim _{\varepsilon \rightarrow 0} \delta_{\varepsilon}(x)=\delta(x)
$$

Note that for $t \leqq \delta_{\varepsilon}(x)$

$$
S_{t}^{\varepsilon} x=x^{\varepsilon}(t)=S_{t} x=x(t) .
$$

Set

$$
\varphi(x)=\sum_{i<j} g\left(x_{i}, x_{j}\right)
$$

and define

$$
\varphi\left(S_{\delta(x)} x\right)=\lim _{\varepsilon \rightarrow 0} \inf \left(S_{\delta_{\varepsilon}(x)} x\right) .
$$


Then by Fatou's lemma and with $\dot{\varphi}=\frac{d}{d t} \varphi$

$$
\begin{aligned}
\int \lambda(d x)\left|\varphi\left(S_{\delta(x)} x\right)\right| & \leqq \int \lambda(d x)|\varphi(x)|+\int \lambda(d x) \liminf _{\varepsilon \rightarrow 0} \int_{0}^{\delta_{\varepsilon}(x)} d t\left|\dot{\varphi}\left(S_{t} x\right)\right| \\
& \leqq \int \lambda(d x)|\varphi(x)|+\liminf _{\varepsilon \rightarrow 0} \int \lambda(d x) \int_{0}^{\delta_{\varepsilon}(x)} d t \mid \dot{\varphi}\left(S_{t}(x) \mid\right.
\end{aligned}
$$

But for $t \leqq \delta_{\varepsilon}(x)$ we obtain in view of (1.1), (1.2), and (2.12)

$$
\begin{aligned}
\dot{\varphi}\left(S_{t} x\right) & =\frac{d}{d t} \varphi(x(t))=\sum_{k=1}^{N}\left(\nabla_{x_{k}(t)} \varphi\right)(x(t)) \cdot \dot{x}_{k}(t) \\
& =\sum_{k=1}^{N} \sum_{i<j}^{N}\left(\nabla_{x_{k}(t)} g\left(x_{i}(t), x_{j}(t)\right)\right) \cdot \sum_{\substack{l=1 \\
l \neq k}}^{N} \gamma_{l} K\left(x_{k}(t), x_{l}(t)\right) \\
& =\sum_{k=1}^{N} \sum_{\substack{j=1 \\
j \neq k}}^{N}\left(\nabla_{x_{k}(t)} g\left(x_{k}(t), x_{j}(t)\right)\right) \cdot \sum_{\substack{l=1 \\
l \neq k}}^{N} \gamma_{l} \nabla_{x_{k}(t)}^{\perp} g\left(x_{k}(t), x_{l}(t)\right) \\
& =\sum_{k=1}^{N} \sum_{\substack{j=1 \\
j \neq k}}^{N} \sum_{\substack{l=1 \\
l \neq k \\
l \neq j}}^{N} \gamma_{l} \nabla_{x_{k}(t)} g\left(x_{k}(t), x_{j}(t)\right) \cdot \nabla_{x_{k}(t)}^{\perp} g\left(x_{k}(t), x_{l}(t)\right),
\end{aligned}
$$

where we used that

$$
\nabla_{x_{k}(t)} g\left(x_{k}(t), x_{j}(t)\right) \cdot \nabla_{x_{k}(t)}^{\perp} g\left(x_{k}(t), x_{l}(t)\right)=0, \quad \text { if } \quad j=1 .
$$

Thus by virtue of (2.1) for $t \leqq \delta_{\varepsilon}(x)$ and some constant $\tilde{C}$

$$
\left|\dot{\varphi}\left(S_{t} x\right)\right| \leqq \tilde{C} \sum_{k=1}^{N} \sum_{\substack{j=1 \\ j \neq k}}^{N} \sum_{\substack{l=1 \\ l \neq k \\ l \neq j}}^{N} \frac{1}{\left|x_{k}(t)-x_{j}(t)\right|} \frac{1}{\left|x_{k}(t)-x_{l}(t)\right|}=h\left(S_{t} x\right) .
$$

Remark. Note that because of the cancellation (2.15) we find the bound (2.16) with the function $h \in L_{1}\left(\bar{T}^{2 N}, \lambda(d x)\right)$.

We now employ (2.11) to obtain for $t \leqq \delta_{\varepsilon}(x)$ from (2.16)

$$
\left|\dot{\varphi}\left(S_{t} x\right)\right|=\left|\dot{\varphi}\left(S_{t}^{\varepsilon} x\right)\right| \leqq h\left(S_{t}^{\varepsilon} x\right) .
$$

Inserting this in (2.14) yields

$$
\int \lambda(d x)\left|\varphi\left(S_{\delta(x)} x\right)\right| \leqq \int \lambda(d x)|\varphi(x)|+\liminf _{\varepsilon \rightarrow 0} \int \lambda(d x) \int_{0}^{\delta_{\varepsilon}(x)} d t h\left(S_{t}^{\varepsilon} x\right) .
$$

Observe that

$$
\int_{0}^{\delta_{\varepsilon}(x)} d t h\left(S_{t}^{\varepsilon} x\right) \leqq \int_{0}^{T} d t h\left(S_{t}^{\varepsilon} x\right)
$$


to conclude with Fubini's theorem and the fact that $S_{t}^{\varepsilon}$ is measure-preserving

$$
\begin{aligned}
\int \lambda(d x)\left|\varphi\left(S_{\delta(x)} x\right)\right| & \leqq \int \lambda(d x)|\varphi(x)|+\liminf _{\varepsilon \rightarrow 0} \int_{0}^{T} d t \int \lambda(d x) h\left(S_{t}^{\varepsilon} x\right) \\
& \leqq \int \lambda(d x)|\varphi(x)|+\int_{0}^{T} d t \int \lambda(d x) h(x) \\
& =\lambda(|\varphi|)+T \lambda(h) .
\end{aligned}
$$

Since $h \in L_{1}\left(\bar{T}^{2 N}, \lambda\right)$ we conclude that there exists a $\lambda$-null set $N_{T}$ such that for all $x \in \bar{T}^{2 N} \backslash N_{T}, \delta(x)=T$ and for $t \leqq T$

$$
\left|\varphi\left(S_{t} x\right)\right|<\infty .
$$

This proves the first part of Theorem 2.1. Inequality (2.7) is now a consequence of the estimates

$$
\lambda\left(\left\{x \mid d_{T}(x)<\varepsilon\right\}\right) \leqq \lambda\left(\left\{x \mid \sup _{0 \leqq t \leqq T} \varphi\left(S_{t} x\right)>-\ln \varepsilon\right\}\right)
$$

and

$$
\int \lambda(d x) \sup _{0 \leqq t \leqq T}\left|\varphi\left(S_{t} x\right)\right| \leqq A(1+T)
$$

which is obtained by essentially repeating the above steps, but using the fact that $S_{t}, 0 \leqq t \leqq T$, is measure-preserving. This follows from the previous part. Then (2.7) is Chebychev's inequality.

As a corollary of the above theorem we have

Theorem 2.2. There exists a set $M \subset \bar{T}^{2 N}$ such that $\lambda(M)=0$ and a one parameter group of $\lambda$-preserving transformations $S_{t}: \bar{T}^{2 N} \backslash M \rightarrow \bar{T}^{2 N} \backslash M$ satisfying (1.1). Moreover if $x \notin M, S_{t} x$ is the only solution of (1.1).

Proof. Obviously Theorem 2.1 holds replacing $T$ by $-T$. Defining $M=\bigcup_{n=-\infty}^{\infty} N_{n}$ we have $\lambda(M)=0$. The flow $S_{t}$ restricted to $\bar{T}^{2 N} \backslash M$ has all the desired properties. Furthermore for any $x \notin M$ and $T>0$ there exists an $\varepsilon>0$ such that $S_{t} x=S_{t}^{\varepsilon} x$ if $t \leqq T$. Let $\tilde{S}_{t} x$ be another solution of (1.1). Then there exists a first time $0<\hat{t}<T$ such that

$$
\left|\hat{S}_{\hat{t}} x_{i}-\tilde{S}_{\hat{t}} x_{j}\right| \leqq \varepsilon
$$

for some $i \neq j$; otherwise $\tilde{S}_{t} x=S_{t}^{\varepsilon} x=S_{t} x$ for all $t \leqq T$. But for $t<\hat{t}$

$$
\left|\tilde{S}_{t} x_{i}-\tilde{S}_{t} x_{j}\right|>\varepsilon
$$

for all $i \neq j$, and thus $\tilde{S}_{t} x=S_{t}^{\varepsilon} x=S_{t} x$ which extends to all $t \leqq T$ by the uniqueness of the solution $S_{t}^{\varepsilon} x$.

The same result can be proved with minor modifications for the case of a bounded domain $D$ with smooth boundary $\partial D$ : The Green's function $g$ is a fundamental solution of the Poisson equation with zero boundary conditions, and the local divergence of $g$ is the same as for the Green's function corresponding to periodic boundary conditions and thus our analysis applies for this case. 
Our proof certainly fails in the case of an unbounded domain. However using compactness methods one can define a global flow satisfying a weak form of Eq. (1.1). But this is not very satisfactory since one does not know whether collapses may be excluded with probability one.

\section{Stochastic Evolution}

In this section we establish the existence and uniqueness of the solution of the system of stochastic differential equations

$$
d x_{i}(t)=\sum_{\substack{j=1 \\ j \neq i}}^{N} \gamma_{j} K\left(x_{i}(t), x_{j}(t)\right) d t+\beta d W_{i}(t), \quad \beta>0, \quad i, j=1, \ldots, N
$$

for $\lambda$-almost all initial conditions $x=\left(x_{1}, \ldots, x_{N}\right) \in \bar{T}^{2 N}$.

Here $W_{i}(t), i=1, \ldots, N$ denote mutually independent standard two dimensional Brownian motions, defined on some probability space $(\Omega, \mathscr{F}, P)$. As above, $K(x, y)$ denotes the vector, field on the torus $\bar{T}^{2}$ defined in (1.2) by means of the Green's function $g$ with periodic boundary conditions. Since the Wiener process on the torus is the standard Wiener process (Brownian motion) modulus $\bar{T}^{2}$, a solution of (3.1) describes a process with continuous trajectories on the torus. As in the previous section we introduce the $\varepsilon$-dynamics

$$
d x_{i}^{\varepsilon}(t)=\sum_{\substack{j=1 \\ j \neq i}}^{N} \gamma_{j} K_{\varepsilon}\left(x_{i}^{\varepsilon}(t), x_{j}^{\varepsilon}(t)\right) d t+\beta d W_{i}(t)
$$

where $K_{\varepsilon}$ is defined by (2.1) and (2.2). For all $\varepsilon>0, K_{\varepsilon} \in C^{1}\left(\bar{T}^{2}\right)$ and hence there exists a unique diffusion process denoted by $x^{\varepsilon}(t, x)=\left(x_{1}^{\varepsilon}(t, x), \ldots, x_{N}^{\varepsilon}(t, x)\right)$ starting almost surely from $x \in \bar{T}^{2 N}$ at time zero and governed by (3.2).

Let $S_{t}$ be the Markov semigroup associated to this process, i.e. for $f \in L_{1}\left(\bar{T}^{2 N}\right)$

$$
\left(S_{t}^{\varepsilon} f\right)(x)=E\left(f\left(x^{\varepsilon}(t, x)\right)\right),
$$

where $E$ denotes the expectation with respect to $(\Omega, \mathscr{F}, P)$.

The following lemma states the conservation of the Lebesgue measure under the action of $S_{t}$.

Lemma 3.1. Let $f \in C^{2}\left(\bar{T}^{2 N}\right)$. Then for any $\varepsilon>0$

$$
\int \lambda(d x)\left(S_{t}^{\varepsilon} f\right)(x)=\lambda(f) .
$$

Proof. The generator of $S_{t}^{\varepsilon}$ is

$$
\sum_{i=1}^{N}\left\{\sum_{\substack{j=1 \\ j \neq i}}^{N} K\left(x_{i}, x_{j}\right) \cdot \nabla_{i}+\frac{1}{2} \beta^{2} \Delta_{i}\right\}
$$

By direct computation one proves that

$$
\frac{d}{d t} \int \lambda(d x)\left(S_{t}^{\varepsilon} f\right)(x)=0, \text { for all } f \in C^{2}\left(\bar{T}^{2 N}\right) .
$$


Let $\tau$ and $\delta$ be defined as in (2.4), (2.6). Note that they are random variables on $\bar{T}^{2 N} \times \Omega$. For $\hat{t}<\delta$ there exists an unique solution of (3.1) with initial value $x$ which we denote by $x(t, x), t \leqq \hat{t}$.

As before we want to prove

Theorem 3.1. For any $T>0, \delta=T \lambda \times P$ a.e.

Proof. Let $\varphi$ be defined as in (2.12). Using (3.1) we write down the stochastic differential of $\varphi$ :

$$
d \varphi(t)=\dot{\varphi}(t) d t+\sum_{i=1}^{N} \nabla_{i} \varphi \cdot d W_{i}(t) .
$$

The following argument is adapted from McKean [5] (Sect. 2.5 and Problem 7). Note that for all $x \in \bar{T}^{2 N}$ and $t \leqq \hat{t}$

$$
\Phi(t)=\int_{0}^{t}\left(\sum_{i=1}^{N}\left(\nabla_{i} \varphi\right)(s)\right)^{2} d s<\infty \quad P \text { a.e. }
$$

Then with

$$
\Phi^{-1}(t)=\min (s \geqq 0 \mid \Phi(s)=t)
$$

we have that

$$
B(t)=\int_{0}^{\Phi-1}(t) \sum_{i=1}^{N} \nabla_{i} \varphi \cdot d W_{i}(s)
$$

is a one dimensional Brownian motion up to $\Phi(\delta) \leqq \infty$ (see [5], p. 29). Hence from (3.5) we obtain in the sense of (2.13)

$$
\varphi(\delta)=\int_{0}^{\delta} \dot{\varphi} d t+B(\Phi(\delta))+\varphi(0) .
$$

Suppose we show

$$
\left|\varphi(0)+\int_{0}^{\delta} d t \dot{\varphi}\right|<\infty \quad \lambda \times P \text { a.e. }
$$

Then if $\delta<T \lim _{t \rightarrow \delta} \varphi(t)=\infty$ and hence $\lim _{t \rightarrow \delta} B(\Phi(t))=\infty$, which is impossible for $B(t)$ being a Brownian motion in either of the cases $\lim _{t \rightarrow \delta} \Phi(t)=\infty$ or $\lim _{t \rightarrow \delta} \Phi(t)<\infty$.

Thus it remains to establish (3.9), which is a consequence of the deterministic analogue. Defining $\tau_{\varepsilon}$ and $\delta_{\varepsilon}$ as in (2.8) and (2.9), we may proceed as in the deterministic case, replacing $\lambda$ by $\lambda \times P$ and using Lemma 3.1 to obtain

$$
\begin{aligned}
& \int \lambda(d x) \int P(d \omega) \int_{0}^{\delta(x, \omega)} d t|\dot{\varphi}(x(t, x, \omega))| \\
& \quad \leqq \liminf _{\varepsilon \rightarrow 0} \int \lambda(d x) \int P(d \omega) \int_{0}^{\delta_{\varepsilon}(x, \omega)} d t|\dot{\varphi}(x(t, x, \omega))| \leqq T \lambda(h)<\infty,
\end{aligned}
$$

where $h$ is defined as in (2.16). 
In complete analogy to the deterministic case one has the following corollary:

Theorem 3.2. For $\lambda$-almost all $x \in \bar{T}^{2 N}$, there exists an unique solution $x(t, x)$ $=\left(x_{1}\left(t, x, \ldots, x_{N}(t, x)\right), x_{i}(t, x) \in \bar{T}^{2}\right.$, starting from $x \in \bar{T}^{2 N}$, of the stochastic differential equation (3.1).

Remark. The above result is still valid if the initial value $x \in \bar{T}^{2 N}$ is $\lambda$-absolutely continuously distributed with a bounded density $\varrho$.

Acknowledgements. One of us (M.P.) thanks the Physics Department of Princeton University for the hospitality and the support given during the period in which this work was done. Also valuable discussions with M. Aizenman and H. Spohn are acknowledged. The co-author wishes to express his gratitude to S. Albeverio and D. Merlini for introducing him to the subject, and is grateful for helpful discussions with S. Goldstein.

\section{References}

1. Kirchhoff, G.: Vorlesungen über Mathematische Physik, 3rd, ed., Vol. 1, p. 257. Leipzig: Teubner 1883

2. Marchioro, C., Pulvirenti, M.: Hydrodynamic in two dimensions and vortex theory. Commun. Math. Phys. 84, 483-503 (1982)

3. Aref, H.: Phys. Fluids 22, 393 (1979)

4. Aizenman, M.: Duke Math. J. 45, 809 (1978)

5. McKean, H.P.: Stochastic Integrals. New York: Academic Press 1969

Communicated by J. Lebowitz

Received December 8, 1981; in revised form February 2, 1982 
